\title{
Biocompatible bacteria@Au composites for application in the photothermal destruction of cancer cells $\dagger$
}

\author{
Wen-Shuo Kuo, ${ }^{a}$ Ching-Ming Wu, ${ }^{b}$ Zih-Syuan Yang, ${ }^{b}$ Szu-Yu Chen, ${ }^{c}$ \\ Cheng-Ying Chen, ${ }^{c}$ Chih-Chia Huang, ${ }^{a}$ Wei-Ming Li, ${ }^{a}$ Chi-Kuang Sun ${ }^{c}$ and \\ Chen-Sheng Yeh $* a$
}

Received (in Cambridge, UK) 27th May 2008, Accepted 30th July 2008

First published as an Advance Article on the web 20th August 2008

DOI: $10.1039 /$ b808871c

\begin{abstract}
We have successfully transformed the infectious $E$. coli bacteria into biocompatible bacteria@Au composites for photothermal therapy.
\end{abstract}

The near-infrared (NIR) region, where tissue transmission is optimal due to low scattering and energy absorption, provides maximum irradiation penetration through tissue. For NIR light absorbing gold-based materials, gold nanostructures such as gold nanoshells coated onto silica spheres, gold nanorods, and gold nanocages have attracted a great deal of attention in the photothermal treatment of tumor cells. ${ }^{1-3}$ These studies suggest that this new class of bioconjugatedand immuno-gold derived nanostructures with good biocompatibility can serve as noninvasive and effective photothermal therapeutic agents for cancer treatment.

The interaction of gold with biological systems has been a topic of interest in widely divergent fields. For example, Abhilash Sugunan and co-workers reported the use of fungi as templates for assembly of gold nanoparticles (NPs) to form microwires. ${ }^{4}$ Radloff et al. studied and applied the Chilo iridescent virus to form gold nanoshells using the metallodielectric approach. ${ }^{5}$ Previous studies have shown the deposition of gold on bacteria from the interaction of bacteria with $\mathrm{HAuCl}_{4}$. Bacillus subtilis bacteria can reduce $\mathrm{Au}^{3+}$ to $\mathrm{Au}^{0}$, but the mechanisms for $\mathrm{Au}^{3+}$ reduction are still not clear. ${ }^{6}$ Integrating biology into materials research to bring forward significant advances in microfabrication resulting in functional structures at the microscale is still a challenge. Inspired by nature, we have successfully converted infectious bacteria into a biocompatible photothermal platform which transforms the microorganisms into a weapon for anti-cancer therapy through the combination of biological microorganisms with the optical properties of gold NPs. To take advantage of the self-nucleation of forming gold nanoshells, the aggregated gold NPs coating on bacteria were conjugated with anti-

${ }^{a}$ Department of Chemistry, National Cheng Kung University, Tainan, 701 Taiwan.E-mail: csyeh@mail.ncku.edu.tw

${ }^{b}$ Department of Cell Biology \& Anatomy, National Cheng Kung University, Tainan, 701 Taiwan

${ }^{c}$ Graduate Institute of Photonics and Optoelectronics, Research Center for Applied Sciences, Academia Sinica, Taipei, 115 Taiwan

$\dagger$ Electronic supplementary information (ESI) available: Experimental section, genotypes and average sizes of different strains of E. coli, TEM, EDX, XRD, UV-visible spectra, cytotoxicity assay, temperature dependence of irradiation time, photothermal efficiency and THG movies. See DOI: 10.1039/b808871c epidermal growth factor receptor (anti-EGFR) antibodies to destroy cancerous cells that overexpress $\mathrm{EGFR}^{3}$ on the cell surface.

In this study, we selected gram-negative bacteria of Escherichia coli (E. coli) as our experimental template. Three different strains of gram-negative bacteria, DH5 $\alpha$, TOPO10, and JM109, were used in the study. Their genotypes are displayed in Table S1 (see Supporting Information $\dagger$ ). The sizes of the three different strains of bacteria range from 1.50 to $3.16 \mu \mathrm{m}$ in length and from 0.37 to $1.18 \mu \mathrm{m}$ in width calculated from 300 counts of bacteria (see Table S2 in Supporting Information $\dagger$ ). We used two methods to prepare the gold nanoshells. In method 1 , we used $5 \mathrm{mM}$ of $\mathrm{HAuCl}_{4}$ solution to incubate bacteria for $32 \mathrm{~h}$. The $\mathrm{AuCl}_{4}{ }^{-}$ions were reduced to form gold NPs and were deposited on the bacterial surface. In method 2, we followed the procedure of Graf et al. ${ }^{7}$ In their method, the gold growing solutions $\mathrm{HAuCl}_{4}$ and $\mathrm{K}_{2} \mathrm{CO}_{3}$ were mixed first to produce $\mathrm{Au}(\mathrm{OH})_{x} \mathrm{Cl}_{y}{ }^{-}$. (The mechanisms are $\mathrm{K}_{2} \mathrm{CO}_{3}+\mathrm{H}_{2} \mathrm{O} \rightarrow \mathrm{HCO}_{3}{ }^{-}+\mathrm{HAuCl}_{4} \rightarrow$ $\left.\mathrm{Au}(\mathrm{OH})_{x} \mathrm{Cl}_{y}{ }^{-}, x+y=4\right)$. The $\mathrm{Au}(\mathrm{OH})_{x} \mathrm{Cl}_{y}{ }^{-}$ions were then reduced to gold NPs by adding a reductant, i.e. $\mathrm{NH}_{2} \mathrm{OH} \cdot \mathrm{HCl}$. However, we added no reducing agent, i.e. $\mathrm{NH}_{2} \mathrm{OH} \cdot \mathrm{HCl}$, to the gold growing solution, but gold NPs still grew and underwent self-aggregation to yield gold nanoshells on the bacteria. At present, the driving force for oxidation and reduction on the bacteria surface remains unknown. It is known that $E$. coli bacteria are composed of peptidoglycan on the cell wall, i.e. glucose, polysaccharides, reducing sugars, and amino acids. Previous studies have suggested that the hydroxyl groups of saccharides and the ionized groups of amino acid residues on the cell wall of the gram-positive bacteria of Bacillus megatherium could serve as sites for $\mathrm{Au}^{3+}$ binding and the aldehyde groups from the reducing sugars act as electron donors to reduce $\mathrm{Au}^{3+}$ to $\mathrm{Au}^{0.8}$.

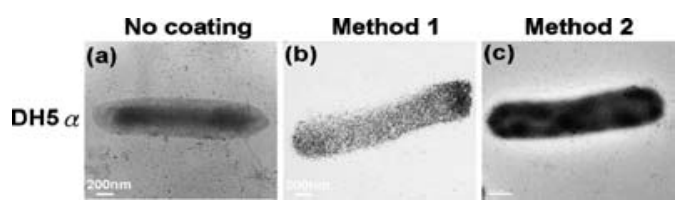

Fig. 1 Bacteria with or without gold nanoshells imaged by TEM. (a) Showing bacteria without gold NPs; bacteria with negative staining using $1 \%$ aqueous sodium phosphotungstate solution. (b) By method 1 and (c) by method 2 showing gold NPs formation for E. coli, DH5 $\alpha$. 
Fig. 1a shows the bare strain of bacteria DH $5 \alpha$ without gold nanoshells. Fig. $1 \mathrm{~b}$ shows that incubation of the bacteria with $\mathrm{HAuCl}_{4}$ solution for $32 \mathrm{~h}$ (method 1 ) resulted in the formation of gold nanoshells composed of gold particles with an average size of $\sim 8 \mathrm{~nm}$. Gold nanoshells consisting of small gold particles with an average size of $\sim 3 \mathrm{~nm}$ were observed on bacteria incubated with the mixture of $\mathrm{HAuCl}_{4}$ and $\mathrm{K}_{2} \mathrm{CO}_{3}$ for $32 \mathrm{~h}$ (method 2), as shown in Fig. 1c. TEM images of multi DH5a@Au composites (methods 1 and 2) are also provided in Supporting Information Fig. S1.† Other different strains, TOPO10 and JM109, were studied and exhibited the same results (see Supporting Information Fig. S2†). EDX and XRD analysis confirmed gold formation using both methods (see Supporting Information Fig. S3 and S4 $\dagger$ ). An ultramicrotoming technique was employed to observe the thicknesses of gold nanoshells for bacteria@Au composites. Fig. 2 indicates that bacteria DH5 $\alpha$ coated with gold nanoshells generated by both methods were approximately $7-8 \mathrm{~nm}$ thick. Similar results were obtained for TOPO10 and JM109 (see Supporting Information Fig. S5 $\dagger$ ).

Third harmonic generation (THG) was utilized to investigate bacteria@Au composites under high spatial resolution. The DH5 $\alpha$ strain was chosen for the following experiments. Since THG is known to be highly sensitive to the interface between two different materials (with different refractive indexes), the morphology of bacteria can be revealed using THG signals. Surprisingly, we found that bacteria remained alive under the nanoshell coating even after two weeks (see Supporting Information Movies 1 and 2 for DH5 $\alpha @$ Au composites generated by method 1 and method 2 , respectively $\dagger$ ). This indicates that the bacteria were able to live in an inorganic environment without nutritional supplements for two weeks.

UV-visible absorbance as a function of incubation time (1-32 h) between DH5 $\alpha$ and gold precursor solutions was monitored for the evolution of gold NPs. The absorbance peaks extending from visible to near-infrared wavelength were contributed from gold NPs (see Supporting Information Fig. S6 $†$ ). The bare bacteria showed no absorption. It is known that highly dispersed spherical gold NPs exhibit an absorbance peak near $520 \mathrm{~nm}$. The characteristic band (surface plasmon band) strongly depends on the size, shape, and degree of aggregation of the gold NPs. As seen in Fig. S6a obtained from method 1 , a broad hump appeared in the range of 500-630 nm with tails toward the IR region, which indicated considerable aggregation in gold NPs (see Supporting Information Fig. S6a $\dagger$ ). As the incubation time increased, the absorptions of the spectra relative to each other increased progressively. TEM observation showed that many gold NPs $(\sim 5.5 \mathrm{~nm})$ could be seen on the bacteria surface in the early

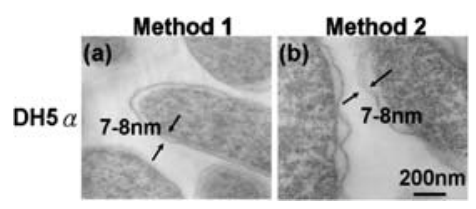

Fig. 2 Ultramicrotomed images for bacteria (DH5 $\alpha$ ) coated with gold nanoshell (a) by method 1 and (b) by method 2 showing 7-8 nm thickness of the gold nanoshell after $32 \mathrm{~h}$ incubation of metal precursors with bacteria. The scale bar is $200 \mathrm{~nm}$. incubation period $\left(\begin{array}{ll}1 & \mathrm{~h}\end{array}\right)$ (see Supporting Information Fig. S7a $\dagger)$. With increasing incubation time $(32 \mathrm{~h})$, larger NPs $(\sim 7.8 \mathrm{~nm})$ with dense aggregation were generated (see Supporting Information Fig. S7b $†$ ). On the other hand, the UV-visible spectra obtained from method 2 (see Supporting Information Fig. S6b $\dagger$ ) showed no gold characteristic peak at an early period of incubation $(\leq 4 \mathrm{~h})$. The contours were merely featureless continua and absorbance curves were toward shorter wavelengths. At a longer incubation time $(\geq 8 \mathrm{~h}$ ), the absorption in the range $500-700 \mathrm{~nm}$ increases, exhibiting a flattening contour. The tail intensifies toward near the IR region when the incubation is prolonged to $32 \mathrm{~h}$. The TEM image showed that rod-like and triangle-like gold NPs with sizes of $\sim 3.0 \mathrm{~nm}$ were produced and a loose state of aggregation for bacteria@Au composites was obtained using method 2 (see Supporting Information Fig. S7c $\dagger$ ). The individual gold NPs $(\sim 3.0 \mathrm{~nm})$ were small, which could account for the featureless absorption (see Supporting Information Fig. S6b $\dagger$ ). The particle size and morphology differentiation might be responsible for the distinct optical properties of the bacteria@Au composites obtained using the two methods.

From the results of the THG experiments (see Supporting Information Movies 1 and $2 \dagger$ ), the bacteria were alive after being covered by the gold nanoshell. It would be worthwhile to study the cytotoxicity of the bacteria $@$ Au composites to mammalian cells. Cell viability experiments were conducted on two cell lines including human keratinocyte nonmalignant cells $(\mathrm{HaCaT})$ and human lung carcinoma malignant cells (A549) using the wellestablished WST-1 assay. ${ }^{9}$ Once again, the DH5 $\alpha$ strain was selected for biocompatibility studies. These DH5 $\alpha @$ Au composites were delivered in a range of dosages $\left(0-100 \mu \mathrm{g} \mathrm{mL}^{-1}\right)$. For DH5 $\alpha$ without gold nanoshells, the cell viability dropped to $40 \%$ when the treating dosage increased to $100 \mu \mathrm{g} \mathrm{mL}^{-1}$. On the other hand, the DH5a@Au composites derived using both methods presented good biocompatibility and showed satisfactory cell viability of more than $80 \%$, even those treated with a high dosage of $100 \mu \mathrm{g} \mathrm{mL} \mathrm{L}^{-1}$ (see Supporting Information Fig. S8 $\dagger$ ). Clearly, the bacteria remained alive after deposition of gold nanoshells and exhibited biocompatibility where gold nanoshells formation seemed to deactivate E. coli bacteria. Further cytotoxicity experiments were performed to extend the incubation time from $24 \mathrm{~h}$ to 10 days using a dosage of $100 \mu \mathrm{g} \mathrm{mL} \mathrm{L}^{-1}$. The cell viability (\%) was evaluated daily for each group. The cells treated with $E$. coli alone or DH5 $\alpha @$ Au composites were divided by the untreated cells, producing cell viability. As seen in Fig. 3, both DH5a@Au composites derived using the two methods had good cell viability of at least $80 \%$ whereas cell viability continued to decrease in

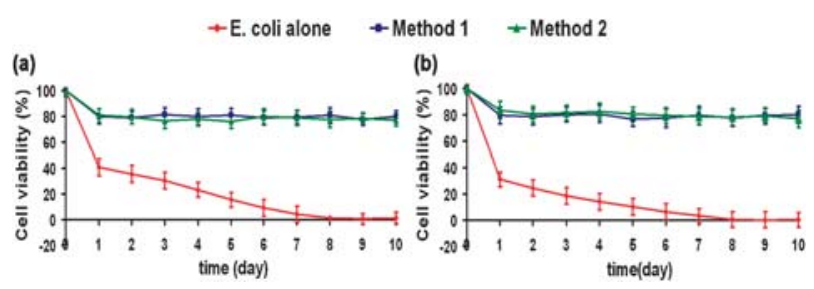

Fig. 3 Cytotoxicity assay (WST-1) of (a) HaCaT and (b) A549 cells treated with DH5 $\alpha$ alone and DH5 $\alpha @$ Au composites at a dosage of $100 \mu \mathrm{g} \mathrm{mL}^{-1}$ for a period of 10 days. 
DH5 $\alpha$ alone as incubation time was prolonged and dropped to zero after the 8th day. It is known that bacteria can trigger a host immune response and can be pathogenic for a wide variety of human diseases attributed to the production of exotoxins and endotoxins. Possibly, gold nanoshells forming on the bacteria surface might have inhibited the production of exotoxins and endotoxins or blocked their contact with the host system. Interestingly, the present results provide an alternative antimicrobial approach to reduce infection and prevent transmission of infectious diseases from bacteria.

As demonstrated in Fig. S6†, DH5 $@ @$ Au composites derived from both methods showed absorption in the NIR region. The biocompatible DH5 $\alpha @$ Au composites might act as efficient photothermal absorbers for destroying cancer cells with a NIR laser. One malignant lung cancer cell line, A549, which overexpresses the epidermal growth factor receptor (EGFR) on the cell surface, was used to study the photothermal effect of the DH5 $\alpha @$ Au composites. For the targeted NIR photothermal therapy, the anti-EGFR antibodies were conjugated with DH5 $\propto$ @u composites through electrostatic interaction. The control A549 cells and the A549 cells treated

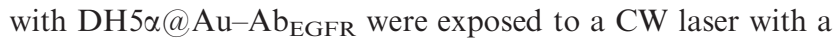
wavelength of $808 \mathrm{~nm}$ (1 mm of laser beam spot) for $6 \mathrm{~min}$ exposure. The laser irradiation dosages were varied from $240 \mathrm{~mW}\left(24 \mathrm{~W} \mathrm{~cm}^{-2}\right)$ to $300 \mathrm{~mW}\left(30 \mathrm{~W} \mathrm{~cm}^{-2}\right)$ (Fig. 4). The cells were incubated with calcein AM and examined by fluorescence microscopy. The colorless calcein AM converted to green fluorescence by enzymatic hydrolysis of ester residues after incorporation into viable cells. In control experiments, no photodestruction of A549 cells was observed. The DH5 $\alpha @ A u-A b_{\text {EGFR }}$ treated A549 cells, on the other hand, revealed a significant loss of cancer cells viability beginning at $260 \mathrm{~mW}\left(26 \mathrm{~W} \mathrm{~cm}^{-2}\right)$, where the area of dead cells lacked green fluorescence. With increasing laser power, the proportion of the void region (area of loss of live cells) spread. This clearly demonstrated that the DH5 $\alpha$ @u $-A b_{\text {EGFR }}$ composites have successfully specifically targeted EGFR overexpressed A549 cells. They effectively absorbed NIR light and converted it to heat up the cell membrane, leading to cell death. We have

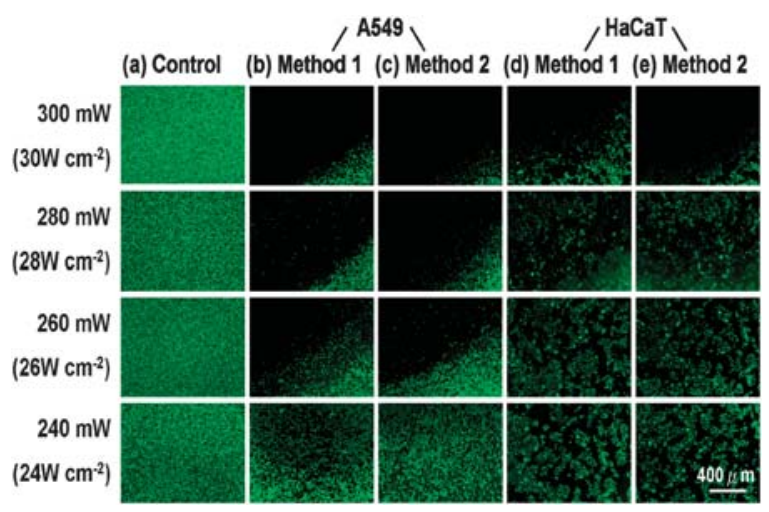

Fig. 4 Fluorescence images show photothermal destruction of malignant A549 and nonmalignant HaCaT cells. The control A549 cells, DH5 $\alpha @ A u-A b_{E G F R}$ treated A549 cells, and DH5 $\alpha @ A u-A b_{E G F R}$ treated HaCaT cells were irradiated by a NIR laser $(808 \mathrm{~nm})$ for 6 min at different laser powers. The cells were stained with calcein AM and live cells exhibited green fluorescence. found that both DH5 $\propto @$ Au composites derived using both methods have the same photothermal effect. Finally, one nonmalignant epithelial cell line, HaCaT, which lacked high expression of the epidermal growth factor receptor (EGFR) on the cell surface, was investigated as well. Because there is less chance of specific antigen-antibody interaction in these benign cells, a smaller amount of DH5 $\alpha @ \mathrm{Au}-\mathrm{Ab} \mathrm{b}_{\mathrm{EGFR}}$ was targeted on $\mathrm{HaCaT}$ cells. As expected, the nonmalignant HaCaT cells suffered photothermal injury at a higher laser power. Cell death occurred after exposure to laser irradiation at $300 \mathrm{~mW}(30 \mathrm{~W}$ $\mathrm{cm}^{-2}$ ). The laser intensities in the range of $240-280 \mathrm{~mW}$ were not sufficient to cause cell injury, and thus cells located in the irradiation area exhibited no loss of viability. The control $\mathrm{HaCaT}$ cells stayed alive after exposure to laser irradiation, which appeared the same as the control A549 cells. To compare the photothermal efficiency of DH5 $@ @$ Au composites prepared by methods 1 and 2, we studied the temperature dependence of irradiation time (without cells). The NIR irradiation induced temperature changed for both composites (see Supporting Information Fig. S9†). The temperature elevated curve as a function of irradiation time from two composites showed a similar trend, but the composite obtained from method 2 presented a slightly better effect of temperature elevation. Additionally, both DH5 $\alpha @ \mathrm{Au}-\mathrm{Ab} \mathrm{b}_{\mathrm{EGFR}}$ composites from methods 1 and $2\left(100 \mu \mathrm{g} \mathrm{mL}^{-1}\right)$ treated with A549 cancer cells at a laser exposure time period of 4-6 min were also studied. It was found that both composites exhibited cell damage at $6 \mathrm{~min}$ (see Supporting Information Fig. S10 $\dagger$ ). Although the temperature dependence of irradiation time (Fig. S9†) showed a slightly better performance for the composites derived from method 2, there is no obvious distinction in the photothermal therapeutic results (Fig. 4 and Fig. S10 $\dagger$ ).

In summary, we have successfully transformed the infectious E. coli bacteria into a biocompatible therapeutic platform. These results might provide an intriguing method to use bacteria and bacteria@Au composites in biomedical applications.

This study was supported by the National Science Council of Taiwan (NSC96-2120-M-006-003) and National Health Research Institute of Taiwan (NHRI-EX97-9201EI).

\section{Notes and references}

1 J. Kim, S. Park, J. E. Lee, S. M. Jin, J. H. Lee, I. S. Lee, I. Yang, J.-S. Kim, S. K. Kim, M.-H. Cho and T. Hyeon, Angew. Chem., Int. Ed., 2006, 45, 7754-7758.

2 L. R. Hirsch, R. J. Stafford, J. A. Bankson, S. R. Sershen, B. Rivera, R. E. Price, J. D. Hazle, N. J. Halas and J. L. West, Proc. Natl. Acad. Sci. U. S. A., 2003, 100, 13549-13554.

3 J. Chen, D. Wang, J. Xi, L. Au, A. Siekkinen, A. Warsen, Z. Y. Li, H. Zhang, Y. Xia and X. Li, Nano Lett., 2007, 7, 1318-1322.

4 A. Sugunan, P. Melin, J. Schnurer, J. G. Hilborn and D. Joydeep, Adv. Mater., 2007, 19, 77-81.

5 C. Radloff, R. A Vaia, J. Brunton, G. T. Bouwer and V. K. Ward, Nano Lett., 2005, 5, 1187-1189.

6 T. J. Beveridge and R. G. E. Murray, J. Bacteriol., 1976, 127, $1502-1518$.

7 C. Graf and A. V. Blaadern, Langmuir, 2002, 18, 524-534.

8 Z.-Y. Liu, J.-M. Wu, B.-Q. Fu, R. Xue, J.-Z. Zhou, Q.-X. Zheng, Y.-Y. Liu and J.-K. Fu, Acta Chim. Sin., 2004, 62, 1829-1834.

9 C.-H. Su, H.-S. Sheu, C.-Y. Lin, Y.-W. Lo, Y.-C. Po, J.-C. Weng, D.-B. Shieh, J.-H. Chen and C.-S. Yeh, J. Am. Chem. Soc., 2007, 129, 2139-2146. 